\title{
Exoplanets with JWST: degeneracy, systematics and how to avoid them
}

\author{
Joanna K. Barstow ${ }^{\mathrm{a}, \mathrm{b}}$, Patrick G. J. Irwin ${ }^{\mathrm{b}}$, Sarah Kendrew ${ }^{\mathrm{c}}$, and Suzanne Aigrain ${ }^{\mathrm{b}}$ \\ ${ }^{a}$ University College London, Department of Physics and Astronomy Gower Street, London, UK \\ ${ }^{b}$ University of Oxford, Department of Physics, Parks Road, Oxford, UK \\ ${ }^{c}$ European Space Agency, Space Telescope Science Institute, 3700 San Martin Drive, Baltimore \\ MD 21218, USA
}

\begin{abstract}
The high sensitivity and broad wavelength coverage of the James Webb Space Telescope will transform the field of exoplanet transit spectroscopy. Transit spectra are inferred from minute, wavelength-dependent variations in the depth of a transit or eclipse as the planet passes in front of or is obscured by its star, and the spectra contain information about the composition, structure and cloudiness of exoplanet atmospheres. Atmospheric retrieval is the preferred technique for extracting information from these spectra, but the process can be confused by astrophysical and instrumental systematic noise.

We present results of retrieval tests based on synthetic, noisy JWST spectra, for clear and cloudy planets and active and inactive stars. We find that the ability to correct for stellar activity is likely to be a limiting factor for cloudy planets, as the effects of unocculted star spots may mimic the presence of a scattering slope due to clouds.

We discuss the pros and cons of the available JWST instrument combinations for transit spectroscopy, and consider the effect of clouds and aerosols on the spectra. Aerosol high in a planet's atmosphere obscures molecular absorption features in transmission, reducing the information content of spectra in wavelength regions where the cloud is optically thick. We discuss the usefulness of particular wavelength regions for identifying the presence of cloud, and suggest strategies for solving the highly-degenerate retrieval problem for these objects.
\end{abstract}

Keywords: radiative transfer, planetary science

\section{INTRODUCTION}

Over the last few years, the number of known exoplanets has increased from a handful to well over 2000. We now have spectroscopic information of a variety of these objects, and it is clear that they represent a diverse range of atmospheric scenarios. ${ }^{1}$ Transit spectroscopy is a key technique for inferring properties of exoplanet atmospheres. When a planet passes in front of (or behind) its parent star, a reduction in the total amount of flux coming from the system can be observed; wavelength-dependent fluctuations in the transit depth encode information about the transmission of starlight through the planet's atmosphere (during transit), or the emergent flux from the planet itself (during eclipse).

The majority of transiting planets for which we have spectroscopic information are hot Jupiters, Jupiter-sized planets in very close orbits around their parent stars. Spectra also exist for a few smaller planets, including the super-Earth GJ 1214b, ${ }^{2}$ which is a world roughly $2.5 \times$ the size of the Earth that is believed to have a gaseous envelope.

Currently, workhorse instruments for transit spectroscopy include Wide Field Camera 3 (WFC3) and the Space Telescope Imaging Spectrograph (STIS) on the Hubble Space Telescope (HST), but such instruments cover only a relatively narrow wavelength region. The upcoming James Webb Space Telescope (JWST) will provide spectral coverage from 0.6 to $\sim 12 \mu \mathrm{m}$, if a system is observed with at least two individual instruments.

Further author information: (Send correspondence to J. K. B.)

J. K B.: E-mail: j.eberhardt@ucl.ac.uk

Space Telescopes and Instrumentation 2016: Optical, Infrared, and Millimeter Wave, edited by Howard A. MacEwen, Giovanni G. Fazio, Makenzie Lystrup, Proc. of SPIE Vol. 9904, 99043P · ( 2016 SPIE · CCC code: 0277-786X/16/\$18 · doi: 10.1117/12.2232543

Proc. of SPIE Vol. $990499043 P-1$ 
This, combined with a primary mirror over $20 \times$ the area of HST, will provide transit spectra of unprecedented information content.

Interpretation of transit spectra relies on modelling and spectral retrieval tools such as NEMESIS. ${ }^{3}$ NEMESIS incorporates a correlated- $\mathrm{k}^{4,5}$ radiative transfer model to simulate the emergent spectrum from the passage of starlight or planetary thermal flux through an atmosphere in transit or eclipse. It also includes an optimal estimation ${ }^{6}$ retrieval algorithm, which allows selection of the best fit atmospheric model for an observed spectrum. Other retrieval methods and algorithms exist that are also likely to be applied to $J W S T$, including $C H I M E R A,{ }^{7}$ TauREX ${ }^{8}$ and others. ${ }^{9,10}$

Following on from previous work by Barstow et al. $(2015)^{11}$ and Greene et al. (2016), ${ }^{12}$ we investigate the possibility of accurately recovering atmospheric properties from primary and secondary transit spectra obtained with JWST. In this work, we use NEMESIS to simulate hot Jupiter spectra as observed with JWST. We then add noise of the expected level for each scenario, and investigate to what extent we could correctly determine the properties of each planet's atmosphere. We discuss the impact of wavelength coverage by comparing observations with the four JWST instruments, and also investigate the effect of star spots on our ability to correctly retrieve the atmospheric state.

\section{CLOUDS IN TRANSMISSION SPECTRA}

Recent observations with HST/STIS and WFC $3^{1}$ indicate that cloud or haze is present on many of the observed hot Jupiters. Given the ubiquity of cloud in the solar system this is not surprising, but cloud presents a challenge for transmission spectra of transiting exoplanets.

A particularly extreme example of this issue is the super Earth GJ 1214b. High precision observations with HST/WFC3 indicate that the planet has a completely flat spectrum, exactly where we would expect a molecular absorption feature due to water vapour. The lack of any such feature implies that the planet has aerosol extending to atmospheric pressures below 1 mbar, which has the effect of obscuring gas absorption features (Figure 1). Because of this, no information is available from the transmission spectrum except the fact that the planet is cloudy.

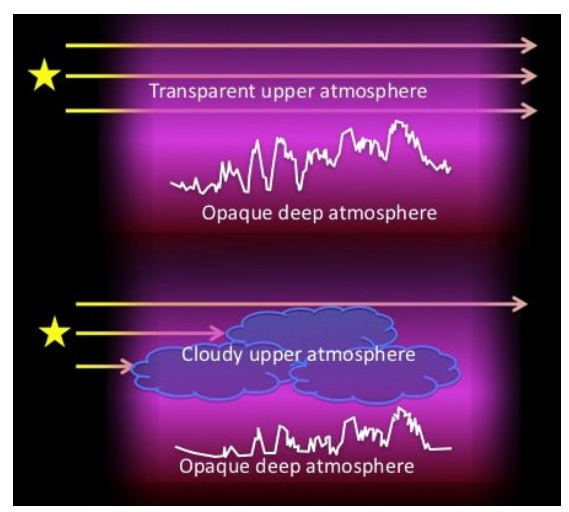

Figure 1. This schematic demonstrates the effect of high clouds on transmission spectra. If any clouds present are deep in the atmosphere, the opacity is dictated by molecular absorbers and the full shapes of absorption features are seen (top). If high cloud is present, however, the wings of absorption features are cut off (bottom). In extreme cases, molecular features can be obscured completely.

We predict that JWST's increased wavelength coverage, and improved spectral resolving power in parts of the spectrum currently only accessible from the ground, will enable planets like GJ 1214b to be further characterised. ${ }^{11}$ In this work, we examine the effect of cloud coverage on spectra of a hot Jupiter orbiting a sun-like star, and discuss implications for the retrieval process. 


\section{MODEL ATMOSPHERES}

In this work, we use the following model atmosphere parameters for a hot Jupiter. We use the same basic planet properties to examine the impact of wavelength coverage in two scenarios, transmission and emission.

We assume a planet of radius $75000 \mathrm{~km}$ and mass $1.8 \times 10^{27} \mathrm{~kg}$. The parent star has the same properties as the Sun, with a radius of $695500 \mathrm{~km}$ and a temperature of $5800 \mathrm{~K}$. We use the Kurucz ${ }^{*}$ spectral model for the Sun to simulate the stellar spectrum.

Gases included in the model are $\mathrm{H}_{2}, \mathrm{He}, \mathrm{H}_{2} \mathrm{O}, \mathrm{CO}_{2}, \mathrm{CO}$ and $\mathrm{CH}_{4}$, as well as $\mathrm{Na}$ and $\mathrm{K}$ for the primary transit case. Sources of all gas absorption data are provided in Table 1

\begin{tabular}{|c|c|}
\hline Gas & Source \\
\hline $\mathrm{H}_{2} \mathrm{O}$ & HITEMP2010 $^{13}$ \\
$\mathrm{CO}_{2}$ & CDSD-1000 $^{14}$ \\
$\mathrm{CO}$ & HITRAN1995 $^{15}$ \\
$\mathrm{CH}_{4}$ & STDS $^{16}$ \\
$\mathrm{H}_{2} / \mathrm{He}$ & Borysow $^{17-21}$ \\
$\mathrm{Na}, \mathrm{K}$ & VALD $^{22}$ \\
\hline
\end{tabular}

Table 1. Sources of gas absorption line data.

The planet is assumed to have a dayside equilibrium temperature of around $1000 \mathrm{~K}$, with the deep atmosphere reaching temperatures of $1800 \mathrm{~K}$. The input temperature profile is shown in comparison with retrieved profiles in Section 5.

We present two sets of tests here. We compare the retrieval of the atmospheric temperature structure and abundances of trace amounts of $\mathrm{H}_{2} \mathrm{O}, \mathrm{CO}_{2}, \mathrm{CO}$ and $\mathrm{CH}_{4}$ from secondary eclipse observations with different combinations of JWST instruments, described in more detail in Section 4. We also investigate the effect of wavelength coverage on our ability to discriminate between different cloud scenarios in primary transit.

\subsection{Dayside retrievals}

The method for the dayside retrievals is based on that presented by Barstow et al. (2015). ${ }^{11}$ We generate 100 versions of a clear-sky hot Jupiter model with variable abundances of the trace gases $\mathrm{H}_{2} \mathrm{O}, \mathrm{CO}_{2}, \mathrm{CO}$ and $\mathrm{CH}_{4}$. We then calculate spectra for these cases, and add appropriate noise for observations with NIRISS, NIRCam, NIRSpec and MIRI-LRS. For more detail on the noise calculation see Section 4. For the secondary transit case, we assume the planet is relatively distant, $250 \mathrm{pc}$ away.

We perform retrievals of temperature as a function of pressure and trace gas volume mixing ratios for each of the 100 models for different combinations of instruments. We compare the quality of the temperature retrieval between different instruments to investigate the effect of wavelength coverage on sensitivity to temperature as a function of altitude. This is an important consideration when deciding which JWST instruments to use for secondary transit observations.

\subsection{Cloud models}

We are especially interested in how well we can constrain cloudy atmospheres in primary transit with JWST. To this end, we add a cloud to our hot Jupiter model. The cloud is based on one of the models used by Barstow et al. $(2014)^{23}$ in our study of the cloudy hot Jupiter HD 189733b. The cloud is assumed to be composed of 0.1 $\mu$ m-radius particles of enstatite $\left(\mathrm{MgSiO}_{3}\right)$, with the extinction cross section calculated using Mie theory from measured refractive indices. ${ }^{24}$ The cloud extends one decade in pressure from 1 to 0.1 mbar.

We test two scenarios for the hot Jupiter in primary transit: a cloud-free model and an optically thin cloudy model. The effect of including cloud can easily be seen on the spectrum (Figure 2). For the noise model in primary transit we choose to assume the planet is somewhat closer, $75 \mathrm{pc}$ away, in order for the spectrum to have sufficient signal-to-noise to discriminate between different cloudy atmosphere scenarios. For this case, we assume the planet is observed using the NIRSpec/MIRI-L instrument combination.

\footnotetext{
${ }^{*}$ http://kurucz.harvard.edu/sun.html
} 


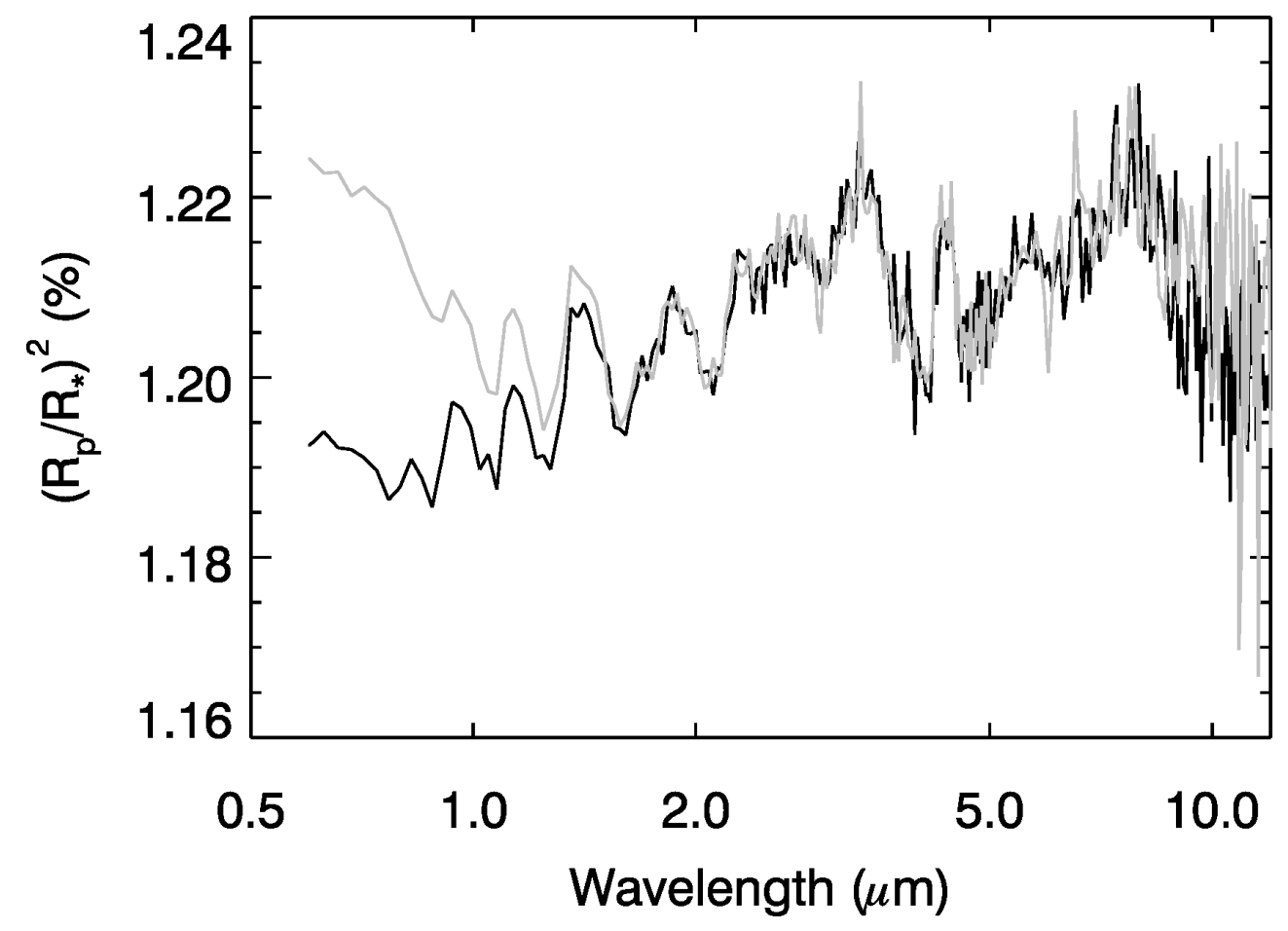

Figure 2. Synthetic spectra for the clear atmosphere case (black) and the cloudy case (grey). The effect of including clouds is obvious at short wavelengths, but we also see differences at longer wavelengths too, demonstrating the importance of broad wavelength coverage for studies of cloudy atmospheres.

We test a range of simple cloud models to retrieve these cloudy spectra. We would not know a priori the particle size or location of the clouds in the atmosphere, or indeed the composition. We compare a series of cloud models that are either purely Rayleigh scattering or completely grey, with a range of cloud top pressures from 100 mbar up to 0.01 mbar. We test two versions of these models, with the cloud either extending from the deep atmosphere all the way to the cloud top, or alternatively with the cloud spanning a decade in pressure below the cloud top. We compare the retrieval results of these models to see if the most suitable cloud model can be determined from JWST primary transit observations.

Retrieved parameters for primary transit are as follows: we recover abundances of trace gases $\mathrm{H}_{2} \mathrm{O}, \mathrm{CO}_{2}, \mathrm{CO}$ and $\mathrm{CH}_{4}$; the cloud opacity; the radius at the 10-bar level in the atmosphere; and a parameterized temperature profile. There is insufficient information in a primary transit spectrum to fully recover the temperature as a function of pressure (see Barstow et al. 2013 ${ }^{25}$ ), but the decrease in temperature in the troposphere has an effect on the variation of the atmospheric scale height in this region, which in turn affects the shape of the transmission spectrum. Therefore, we parameterize the T-p profile as a constant value up to a deep knee pressure (set at 3 bars in this case, which is below the region of the atmosphere to which we are sensitive) which decreases with some variable fractional scale height above the knee pressure. This allows temperature variation in the region of maximum sensitivity to be captured with only two free parameters.

\subsection{Star spots}

We adopt the same method for starspot modelling as that used in our previous work. ${ }^{11}$ We assume the spot contrast for a sun-like star is simply the ratio of blackbodies at the spot and photospheric temperatures. We assume that spots are at $5000 \mathrm{~K}$, whereas the rest of the stellar photosphere is at $5800 \mathrm{~K}$. As in previous work, we assume that the spots cover approximately $3 \%$ of the stellar disk and the planet does not occult any spots during transit. 
The particular focus of the spot modelling in this work is to investigate the effect of starspots on our ability to accurately infer the presence or absence of cloud. McCullough et al. $(2014)^{26}$ suggest that, for large enough out-of-transit spot fractions, it may be possible for unocculted spots to mimic Rayleigh scattering due to the presence of atmospheric aerosol. This is because the spots are cooler than the stellar photosphere, therefore if the planet transits a spot-free region it is blocking a region of the star that is slightly brighter than the average, and the planet appears to be bigger. The effect is more pronounced at short wavelengths where the spot/photosphere contrast is highest, hence the possibility of mimicking clouds.

\section{JWST}

JWST will be a powerful tool for observations of transiting exoplanets. Here, we discuss the different instrument scenarios that may be employed, and the noise models assumed in this work for each.

\subsection{JWST instruments}

JWST has four spectroscopic instruments on board, all of which may be used to great effect for transit spectroscopy. Three of these cover the near-infrared region, between 0.6 and $5 \mu \mathrm{m}$, whilst the fourth, the Mid InfraRed Instrument (MIRI) covers wavelengths longwards of $5 \mu \mathrm{m}$.

A key consideration for exoplanet transit spectroscopy is that a large spectral range should be covered in as few observations as possible. Barstow et al. $(2015)^{11}$ discussed the potential problems of stitching together observations taken at different times over different wavelength ranges if conditions have changed in between the observations. This can be corrected to some extent if instruments have overlapping wavelengths, but observational scenarios should be chosen to avoid excessive stitching where possible.

In the near infrared, the Near InfraRed Imager and Slitless Spectrograph (NIRISS) has spectroscopic capabilities up to $2.5 \mu \mathrm{m}$ with the GR700XD grism. The assumed throughput here is taken from Doyon et al. (2012). ${ }^{27}$ This is now a conservative estimate as an alternative grism with higher throughput may be used. The Near InfraRed Camera (NIRCam) can be used with the F322W2 and F444W grisms, covering the wavelength region between 2.5 and $5 \mu \mathrm{m}$ in two shots. Total throughput for this instrument was estimated to be 0.6 , which is a little higher than current data would suggest; however, the important test of the effect of increasing wavelength coverage is unlikely to be affected.

Together, NIRISS and NIRCam can cover the $0.6-5 \mu \mathrm{m}$ wavelength region in 3 shots. The Near InfraRed Spectrograph (NIRSpec) in prism mode can cover wavelengths from 0.6 to $5 \mu \mathrm{m}$ in a single shot, so might be assumed to be a better choice. However, NIRSpec in prism mode has much more stringent bright target limits than other modes ${ }^{28}$ and also has tight bright limits compared with other near-infrared instruments. Therefore, NIRSpec might be appropriate for faint targets, but for bright targets NIRISS and NIRCam is likely to be the preferred combination.

The MIRI Low Resolution Spectrometer (LRS) covers 5 to around $12 \mu \mathrm{m}$ in one shot. Noise estimates for MIRI and NIRSpec are as used by Barstow et al. (2015). ${ }^{11,29,30}$ MIRI also has a second mode, the Medium Resolution Spectrometer, which covers the wavelength range from 5 to $28 \mu \mathrm{m}$ split across four spectroscopic channels. Each of three observing modes covers $1 / 3$ of each spectroscopic channel, therefore the full wavelength range can be achieved with three observations. The throughput for the MRS is a factor of three lower at short wavelengths than for the LRS and it decreases significantly longwards of $15 \mu \mathrm{m}$. This, coupled by the requirement for three separate observations to cover the wavelength range, makes it unlikely that the MRS will be used extensively for transit spectroscopy.

\subsection{Noise calculation}

The noise calculation used is the same as that from Barstow et al. (2015). ${ }^{11}$ We reproduce the equation here for convenience. The stellar flux is converted into the number of photons incident on the detector, then the photon noise is calculated by taking the square root and the equation is reversed to give the noise on the measured stellar flux.

$$
n_{\lambda}=I_{\lambda} \pi\left(r_{\star} / D_{\star}\right)^{2}(\lambda / h c)(\lambda / R) A_{e f f} Q \eta t
$$


where $n_{\lambda}$ is the number of photons received for a given wavelength $\lambda, I_{\lambda}$ is the spectral radiance of the stellar signal, $r_{\star}$ is the stellar radius, $D_{\star}$ is the distance to the star, $h$ and $c$ are the Planck constant and speed of light, $R$ is the spectral resolving power, $A_{\text {eff }}$ is the telescope effective area, $Q$ is the detector quantum efficiency, $\eta$ is the the throughput and $t$ is the exposure time.

The noise on the transit depth is then given by the equation $\sqrt{(2) * \sigma_{I} / I}$.

\section{RESULTS AND DISCUSSION}

Here, we show the results of secondary transit retrievals for a cloud-free hot Jupiter and primary transit retrievals for a cloudy hot Jupiter.

\subsection{A cloudy hot Jupiter in primary transit}

Here, we present the results of retrievals for a cloudy hot Jupiter in primary transit. We have retrieved abundances of $\mathrm{H}_{2} \mathrm{O}, \mathrm{CO}_{2}, \mathrm{CO}$ and $\mathrm{CH}_{4}$, a deep temperature and fractional scale height, a multiplying factor on the cloud opacity, and the radius at the 10-bar pressure level. What we are really interested in is whether a) we can accurately recover the abundances of the four trace gases, even in the presence of clouds and b) whether we can place some constraint on the vertical position and likely particle size of the cloud.

First, we test the clear atmosphere synthetic observation, to see if we can confirm that the atmosphere is clear, or whether it can also be represented by cloudy models. Models with some Rayleigh scattering aerosol present can fit the spectra well, but the retrieved optical depths are generally low, which is consistent with only a marginal detection of cloud, as we might expect. Models with grey aerosol, even small amounts, do not fit the spectra as well, simply because the clouds are relatively more opaque at longer wavelengths than the Rayleigh scattering cloud.

For the clear fit with Rayleigh scattering clouds, we examine the effect of including clouds on our ability to accurately recover abundances of molecular absorbers from the spectrum. In general, including clouds in retrievals of a clear atmosphere spectrum introduces degeneracies that result in abundances of molecular species being overestimated. This is because the cloud slightly flattens the spectrum and raises the continuum level, so gas abundances increase to compensate for this effect. Conversely, the radius at the 10-bar pressure level (not shown) is consistently under-retrieved to compensate for the raised continuum level.

$\mathrm{CO}$ is not accurately retrieved in any case, due to its few spectral features that are usually masked by absorption due to other gases. $\mathrm{H}_{2} \mathrm{O}$ and $\mathrm{CH}_{4}$ are retrieved well for the clear atmosphere case, and $\mathrm{CO}_{2}$ (which has a very low abundance in this example) is retrieved correctly to within $2 \sigma$.

Secondly, we test the HD 189733b-based cloudy model. In this case, the only cloud model that produces an adequate fit to the noisy spectrum is a Rayleigh scattering cloud covering a decade in pressure between 1 and 0.1 mbar (Figure 4). This corresponds well to the input case, which is a cloud made of $0.1 \mu \mathrm{m}$-sized particles covering exactly that pressure range. This demonstrates that we can well recover basic cloud properties from primary transit spectra with $J W S T$.

In addition, we also recover the $\mathrm{H}_{2} \mathrm{O}$ and $\mathrm{CH}_{4}$ abundances correctly to within error for this case. The $\mathrm{CO}$ abundance is not well retrieved, which is consistent with the clear atmosphere case, and due to its low abundance $\mathrm{CO}_{2}$ features are masked by the presence of the cloud layer.

\subsection{Effect of starspots on recovered properties}

Barstow et al. (2015) $)^{11}$ test the effect of $5000 \mathrm{~K}$ spots on a sun-like star that cover $3 \%$ of the out-of-transit disc. Unocculted starspots are especially problematic as their signatures don't show up in transit lightcurves, but their presence can introduce a chromatic bias in transmission spectra. ${ }^{31}$ This effect is more pronounced at short wavelengths where the spot/disc contrast is greatest, and therefore has a particularly strong effect on inferences about scattering cloud.

McCullough et al. (2014) ${ }^{26}$ suggest that such effects may be responsible for the apparent presence of cloud on the hot Jupiter HD 189733b found by Pont et al. (2013). ${ }^{31}$ We wanted to test this hypothesis by comparing 


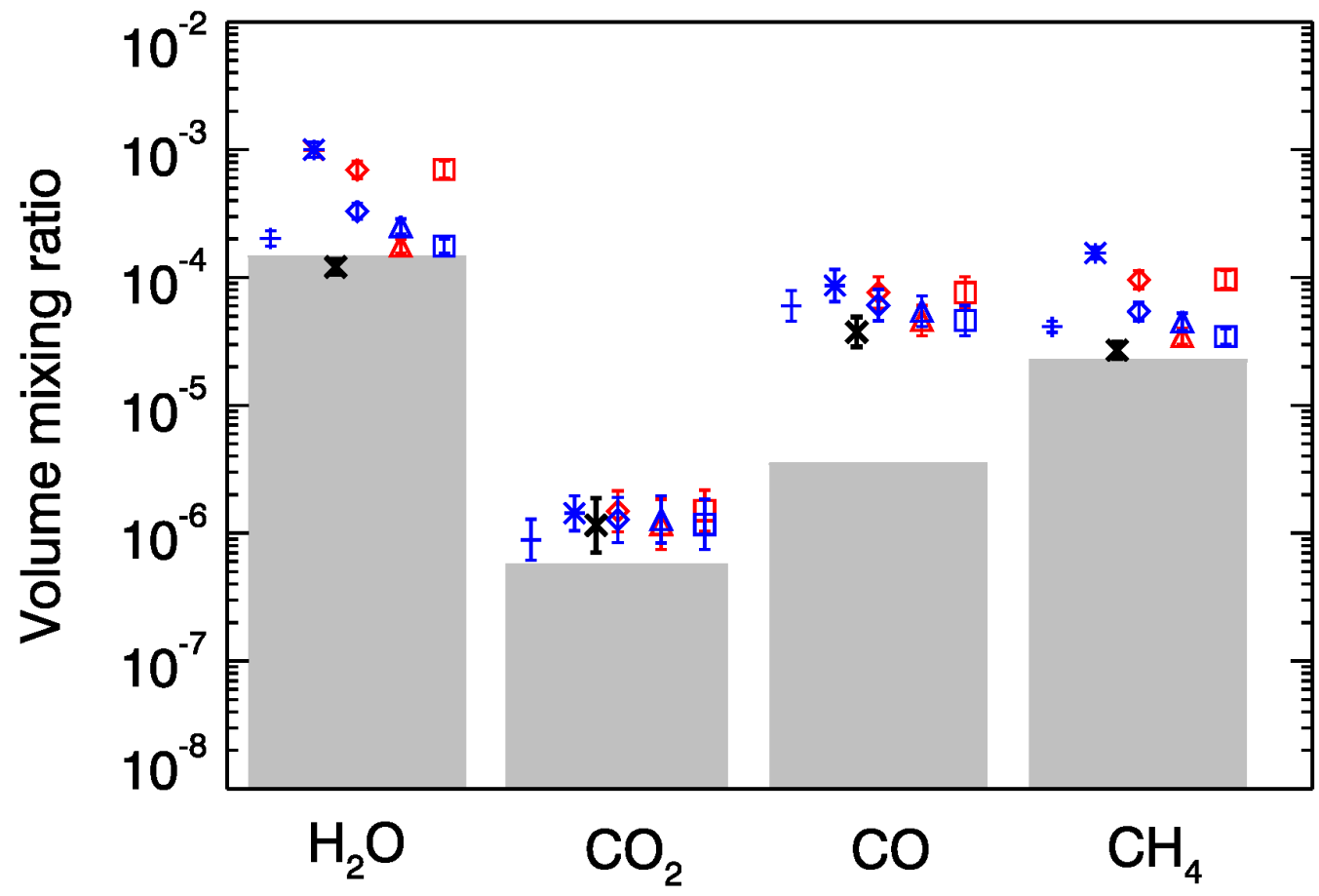

Figure 3. Retrieved gas abundances for a variety of cloud models fit to a synthetic observation of a clear hot Jupiter. The black points are fits with a clear model, the red points are fits with extended cloudy models and blue points are first with decade cloud models. Crosses, asterisks, diamonds, triangles and squares are models with cloud tops at 100, 10, 1, 0.1 and 0.01 mbar. The grey bars show the input values. The a priori abundance for all cases is $10^{-4}$.

retrievals of the starspot-free, clear-sky hot Jupiter case with the same planetary model assuming $3 \%$ out of transit spot coverage.

We did not find any stronger evidence for extended clouds in the retrievals of the spotty case than we did in the spot-free clear-sky case. However, for decade-confined clouds with cloud top pressures at 1, 0.1 and 0.01 mbar we retrieve cloud optical depths a factor of $2-3$ higher for the spotty case. This suggests that spots may indeed masquerade as optically thin cloud, but the detection of molecular features in the infrared should prevent a mistaken retrieval of an opaque cloud layer. There is also slightly increased degeneracy in the retrieval of gas abundances (Figure 5), with $\mathrm{H}_{2} \mathrm{O}$ more substantially over-retrieved, but in general the molecular abundances retrieved are not greatly affected.

We also tested the effect of star spots on retrievals of the cloudy atmosphere case. This turned out to be more problematic as none of the cloud models in our test set produced an adequate fit to the data, since we could not reproduce the steepness of the short wavelength slope. This slope is a produced by a combination of scattering clouds and starspots. It should be noted that a short wavelength slope that is substantially steeper than a $1 / \lambda^{4}$ trend cannot conceivably be produced by scattering clouds, as the Rayleigh scattering limit of $1 / \lambda^{4}$ is the steepest limit, therefore an extremely steep slope could on its own be an indicator of the presence of unocculted starspots. However, disentangling the magnitude of this effect from any cloud signatures present would be impossible without additional information.

In this situation, we would require comprehensive monitoring of the star, as performed by Pont et al. (2013) $)^{31}$ for HD 189733b, to infer the approximate spot coverage at the time of observation. This kind of monitoring, combined with the presence or absence of spot crossing events during transit, would enable the effect of starspots on the spectrum to be estimated, which would substantially improve the quality of the retrieval. 

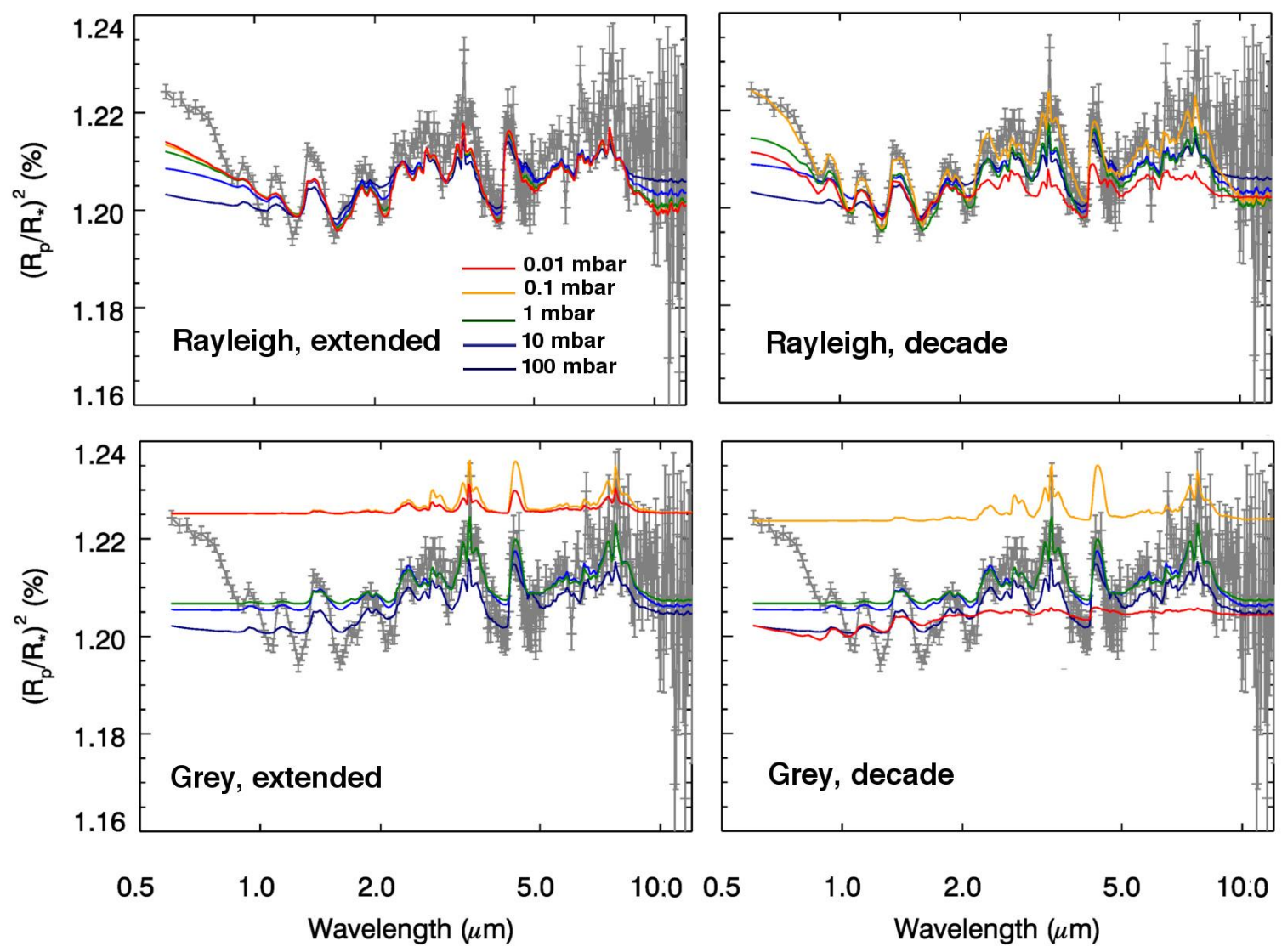

Figure 4. Fits with a variety of cloud models to a synthetic observation of a cloudy hot Jupiter. Only the Rayleigh scattering model with a cloud top at 0.01 mbar, spanning a decade in pressure, fits the synthetic measurement.

\subsection{A clear-sky hot Jupiter in secondary transit for different instrument combinations}

We also present retrieval results for the clear-sky hot Jupiter case in secondary transit, to demonstrate the effect of increasing wavelength coverage on our ability to recover the atmospheric temperature structure.

In Figure 6 we compare the average over 100 temperature retrievals for the following scenarios: NIRISS only (up to $2.5 \mu \mathrm{m}$ ); NIRISS+NIRCam (up to $5 \mu \mathrm{m}$ ); and NIRISS+NIRCam+MIRI-LRS (up to $12 \mu \mathrm{m}$ ). It is clear that using NIRISS alone does not provide sufficient information to constrain the temperature profile. Adding the NIRCam data and extending the wavelength range up to $5 \mu \mathrm{m}$ makes a substantial differenc, providing information about pressures below 0.1 bar. Adding the MIRI-LRS range doesn't add sensitivity to further pressure ranges, but by increasing the coverage it does improve the accuracy and precision of the retrieval.

As indicated by Stevenson et al. (2016), ${ }^{32}$ instrument combinations covering the wavelength range from 0.6 to $12 \mu \mathrm{m}$ are likely to be the standard for comprehensive exoplanet characterisation observations. We demonstrate here that $0.6-5 \mu \mathrm{m}$ coverage is critical, and $0.6-12 \mu \mathrm{m}$ coverage is highly desirable for secondary transit observations.

We also compare the use of NIRISS+NIRCam at short wavelengths versus the use of NIRSpec. The two instrument combinations produce very similar results, with the NIRISS+NIRCam combination producing slightly more accurate temperature retrievals, but with the disadvantage of requiring three observations to cover a wavelength range NIRSpec can obtain in one. 


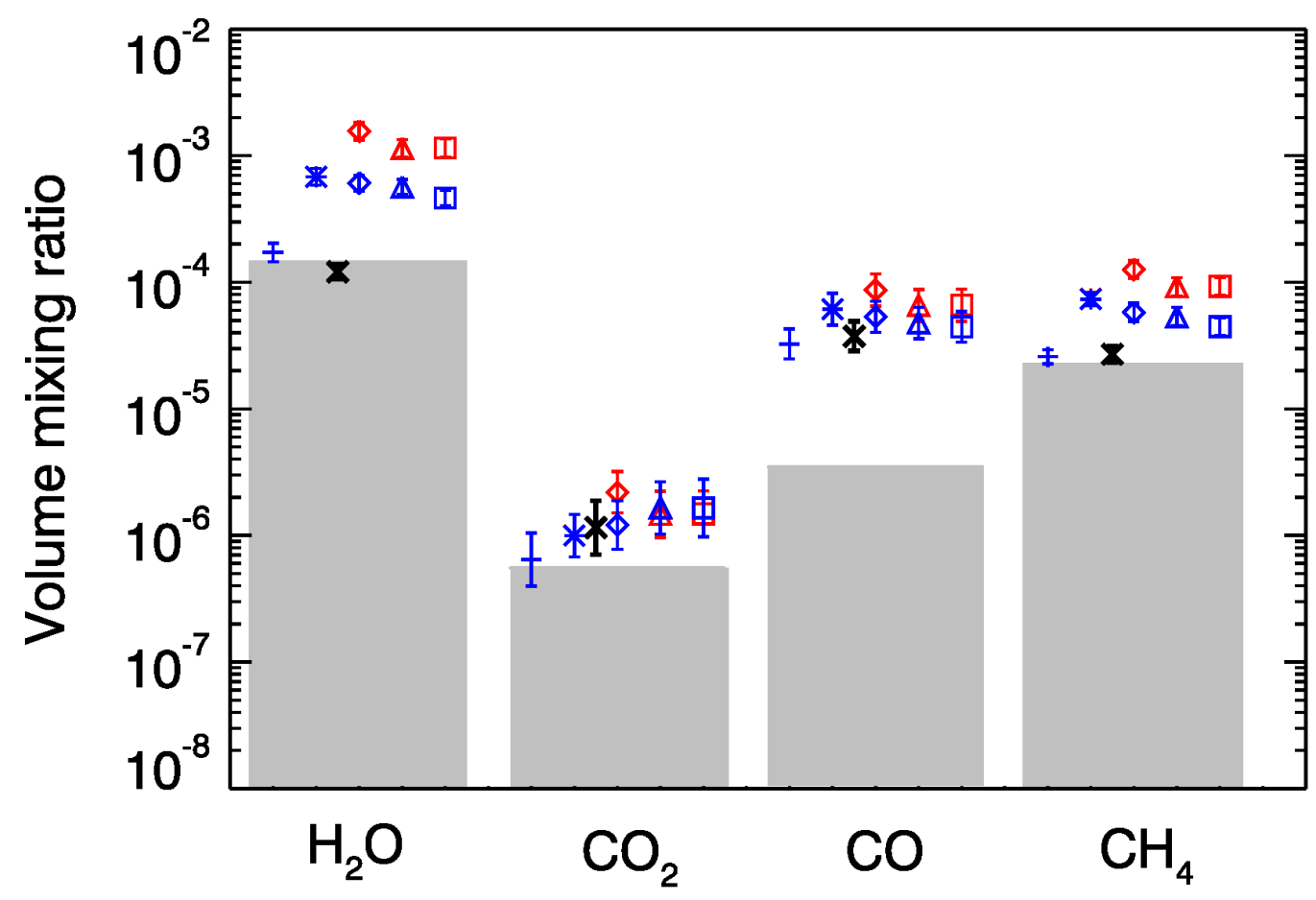

Figure 5. Retrieved gas abundances for a variety of cloud models fit to a synthetic observation of a clear hot Jupiter, assuming $3 \%$ out of transit spot coverage with a temperature contrast of $800 \mathrm{~K}$. The black points are fits with a clear model and no star spots, the red points are fits with extended Rayleigh cloud models and blue points are fits with decade Rayleigh cloud models. Crosses, asterisks, diamonds, triangles and squares are models with cloud tops at 100, 10, 1, 0.1 and 0.01 mbar. The grey bars show the input values. The a priori abundance for all cases is $10^{-4}$.

\section{CONCLUSIONS}

We demonstrate that broad wavelength coverage is important for temperature retrievals from secondary transit observations with JWST. Wavelength coverage from at least $0.6-5 \mu \mathrm{m}$ is critical, with extension up to $12 \mu \mathrm{m}$ adding greater precision and accuracy.

Following on from initial findings by Barstow et al. (2015), ${ }^{11}$ we find that $J W S T$ will be an extremely powerful tool for discriminating between different cloud structures in primary transit. We find that we can unambiguously identify the correct vertical cloud structure and the bulk scattering properties for a hot Jupiter with a simple, single-layer $\mathrm{MgSiO}_{3}$ cloud of $0.1 \mu \mathrm{m}$ particles. We can also produce reasonable retrievals of gas abundances from cloudy and clear primary transit spectra, although we find it difficult to completely rule out the presence of cloud for a clear atmosphere scenario.

The presence of unocculted starspots is likely to be a key complication for JWST observations of transiting exoplanets. Following on from discussion of the effect on molecular abundance retrievals by Barstow et al. (2015), ${ }^{11}$ we have now considered the effect on retrievals of cloud properties. We find that $3 \%$ out-of-transit spot coverage for a sun-like star would not have a significant effect on retrievals of clear atmospheres, but the summed effects of scattering cloud and unocculted starspots can produce apparent 'scattering' slopes at short wavelengths that appear to be unphysically large, causing difficulties with spectral fitting. We recommend monitoring of host stars for key targets to assess the levels of stellar activity and to estimate spot coverage during observations, as performed by Pont et al. (2013). ${ }^{31}$ 


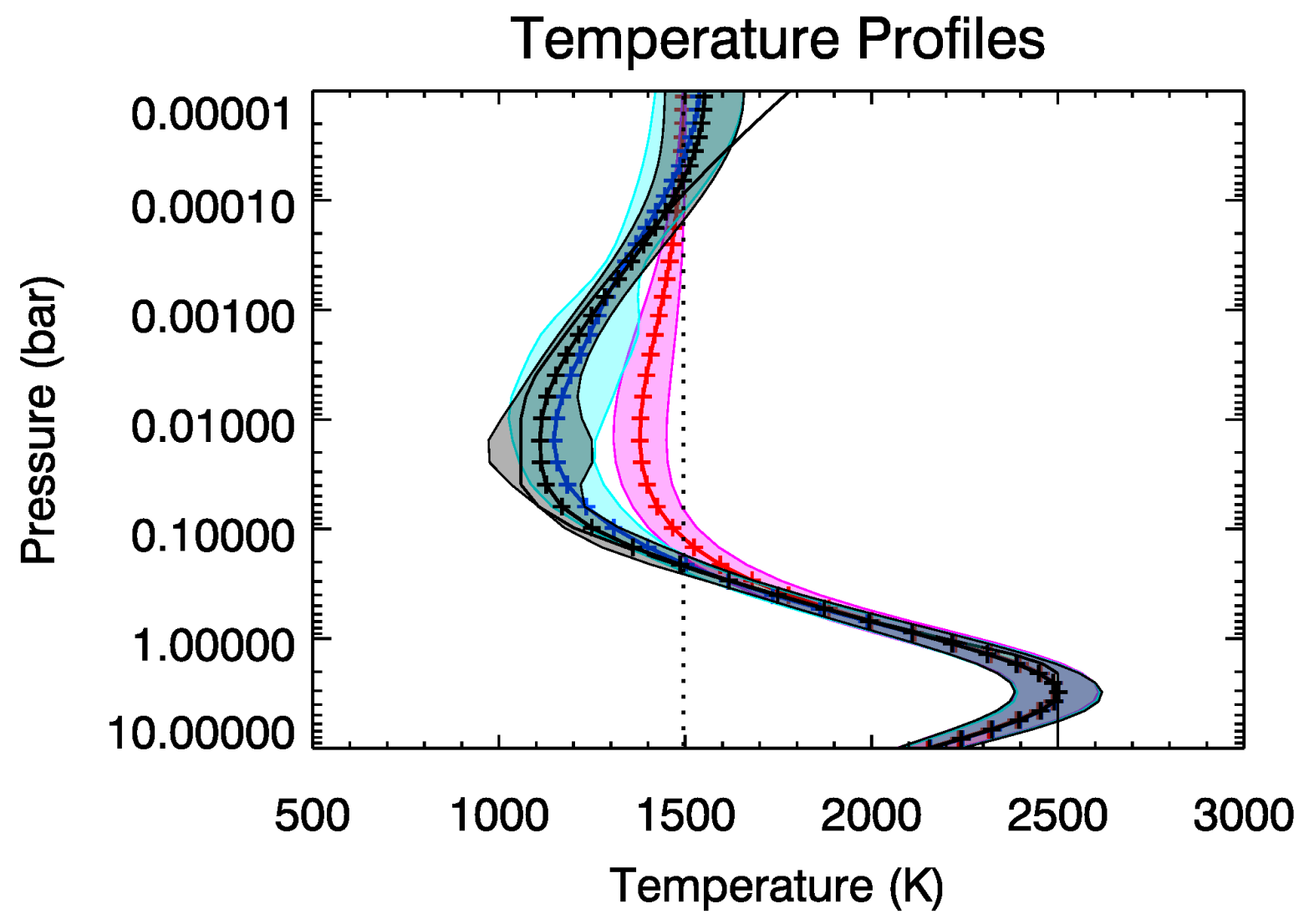

Figure 6. Temperature retrievals over 100 models for three different instrument combinations. For each colour, the thick lines and crosses are the mean retrieved profiles and the shaded regions are the $1 \sigma$ standard deviations across the 100 models. Pink/red = NIRISS only, aqua/blue $=$ NIRISS +NIRCam, and grey $/$ black $=$ NIRISS+NIRCam+MIRI-LRS. The thick solid black line is the input profile and the dashed black line is the temperature prior.

\section{ACKNOWLEDGMENTS}

JKB acknowledges the support of ERC project 617119 (ExoLights) and PGJI acknowledges the support of the Science and Tech- nology Facilities Council.

\section{REFERENCES}

[1] Sing, D. K., Fortney, J. J., Nikolov, N., Wakeford, H. R., Kataria, T., Evans, T. M., Aigrain, S., Ballester, G. E., Burrows, A. S., Deming, D., Désert, J.-M., Gibson, N. P., Henry, G. W., Huitson, C. M., Knutson, H. A., Etangs, A. L. D., Pont, F., Showman, A. P., Vidal-Madjar, A., Williamson, M. H., and Wilson, P. A., "A continuum from clear to cloudy hot-Jupiter exoplanets without primordial water depletion," Nature 529, 59-62 (Jan. 2016).

[2] Charbonneau, D., Berta, Z. K., Irwin, J., Burke, C. J., Nutzman, P., Buchhave, L. A., Lovis, C., Bonfils, X., Latham, D. W., Udry, S., Murray-Clay, R. A., Holman, M. J., Falco, E. E., Winn, J. N., Queloz, D., Pepe, F., Mayor, M., Delfosse, X., and Forveille, T., "A super-Earth transiting a nearby low-mass star," Nature 462, 891-894 (Dec. 2009).

[3] Irwin, P. G. J., Teanby, N. A., de Kok, R., Fletcher, L. N., Howett, C. J. A., Tsang, C. C. C., Wilson, C. F., Calcutt, S. B., Nixon, C. A., and Parrish, P. D., "The NEMESIS planetary atmosphere radiative transfer and retrieval tool," Journal of Quantitative Spectorscopy and Radiative Transfer 109, 1136-1150 (2008). 


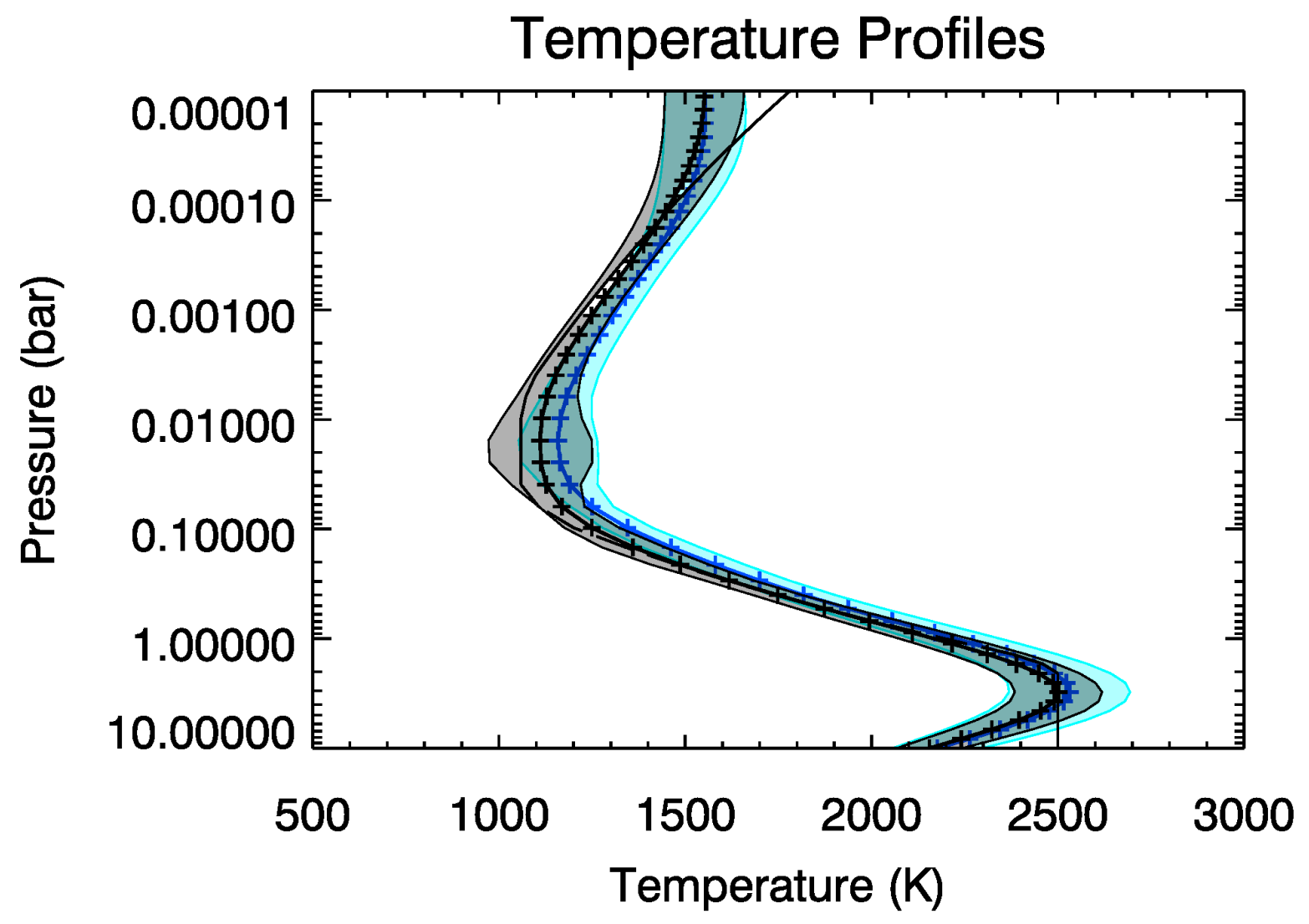

Figure 7. Temperature retrievals over 100 models for NIRISS+NIRCam+MIRI-LRS and NIRSpec+MIRI-LRS. For each colour, the thick lines and crosses are the mean retrieved profiles and the shaded regions are the $1 \sigma$ standard deviations across the 100 models. Aqua/blue $=$ NIRSpec+MIRI-L and grey/black $=$ NIRISS+NIRCam+MIRI-LRS. The thick solid black line is the input profile and the dashed black line is the temperature prior. The NIRISS+NIRCam combination at short wavelengths provides a slightly better retrieval but requires more observations.

[4] Lacis, A. A. and Oinas, V., "A description of the correlated-k distribution method for modelling nongray gaseous absorption, thermal emission, and multiple scattering in vertically inhomogeneous atmospheres," $J$. Geophys. Res. 96, 9027-9064 (1991).

[5] Goody, R. M. and Yung, Y. L., [Atmospheric radiation : theoretical basis] (1989).

[6] Rodgers, C. D., [Inverse Methods for Atmospheric Sounding], World Scientific (2000).

[7] Line, M. R., Knutson, H., Wolf, A., and Yung, Y., "A Systematic Retrieval Analysis of Secondary Eclipse Spectra II: A Uniform Analysis of Eight Planets and Their C to O Ratios," ArXiv e-prints (Sept. 2013).

[8] Waldmann, I. P., "On Signals Faint and Sparse: The ACICA Algorithm for Blind De-trending of Exoplanetary Transits with Low Signal-to-noise," ApJ 780, 23 (Jan. 2014).

[9] Madhusudhan, N. and Seager, S., "A Temperature and Abundance Retrieval Method for Exoplanet Atmospheres," The Astrophysical Journal 707, 24-39 (2009).

[10] Benneke, B. and Seager, S., "Atmospheric Retrieval for Super-Earths: Uniquely Constraining the Atmospheric Composition with Transmission Spectroscopy," The Astrophysical Journal 753, 100 (July 2012).

[11] Barstow, J. K., Aigrain, S., Irwin, P. G. J., Kendrew, S., and Fletcher, L. N., "Transit spectroscopy with James Webb Space Telescope: systematics, starspots and stitching," MNRAS 448, 2546-2561 (Apr. 2015).

[12] Greene, T. P., Line, M. R., Montero, C., Fortney, J. J., Lustig-Yaeger, J., and Luther, K., "Characterizing Transiting Exoplanet Atmospheres with JWST," ApJ 817, 17 (Jan. 2016). 
[13] Rothman, L. S., Gordon, I. E., Barber, R. J., Dothe, H., Gamache, R. R., Goldman, A., Perevalov, V. I., Tashkun, S. A., and Tennyson, J., "HITEMP, the high-temperature molecular spectroscopic database," Journal of Quantitative Spectroscopy and Radiative Transfer 111, 2139-2150 (Oct. 2010).

[14] Tashkun, S. A., Perevalov, V. I., Teffo, J.-L., Bykov, A. D., and Lavrentieva, N. N., "CDSD-1000, the hightemperature carbon dioxide spectroscopic databank," Journal of Quantitative Spectroscopy and Radiative Transfer 82, 165-196 (Nov. 2003).

[15] Rothman, L. S., Wattson, R. B., Gamache, R., Schroeder, J. W., and McCann, A., "HITRAN HAWKS and HITEMP: high-temperature molecular database," in [Society of Photo-Optical Instrumentation Engineers (SPIE) Conference Series], Dainty, J. C., ed., Society of Photo-Optical Instrumentation Engineers (SPIE) Conference Series 2471, 105-111 (June 1995).

[16] Wenger, C. and Champion, J. P., "Spherical Top Data System (STDS) software for the simulation of spherical top spectra.," Journal of Quantitative Spectroscopy and Radiative Transfer 59, 471-480 (May 1998).

[17] Borysow, A. and Frommhold, L., "Collision-induced infrared spectra of H2-He pairs at temperatures from 18 to 7000 K. II - Overtone and hot bands," The Astrophysical Journal 341, 549-555 (June 1989).

[18] Borysow, A., Frommhold, L., and Moraldi, M., "Collision-induced infrared spectra of H2-He pairs involving 0-1 vibrational transitions and temperatures from 18 to $7000 \mathrm{~K}, "$ The Astrophysical Journal 336, 495-503 (Jan. 1989).

[19] Borysow, A. and Frommhold, L., "A new computation of the infrared absorption by H2 pairs in the fundamental band at temperatures from 600 to 5000 K," The Astrophysical Journal Letters 348, L41-L43 (Jan. 1990).

[20] Borysow, A., Jorgensen, U. G., and Zheng, C., "Model atmospheres of cool, low-metallicity stars: the importance of collision-induced absorption.," Astronomy and Astrophysics 324, 185-195 (Aug. 1997).

[21] Borysow, A., "Collision-induced absorption coefficients of $\mathrm{H}_{2}$ pairs at temperatures from $60 \mathrm{~K}$ to $1000 \mathrm{~K}$," Astronomy and Astrophysics 390, 779-782 (Aug. 2002).

[22] Heiter, U., Barklem, P., Fossati, L., Kildiyarova, R., Kochukhov, O., Kupka, F., Obbrugger, M., Piskunov, N., Plez, B., Ryabchikova, T., Stempels, H. C., Stütz, C., and Weiss, W. W., "VALD - an atomic and molecular database for astrophysics," Journal of Physics Conference Series 130, 012011 (Oct. 2008).

[23] Barstow, J. K., Aigrain, S., Irwin, P. G. J., Hackler, T., Fletcher, L. N., Lee, J. M., and Gibson, N. P., "Clouds on the Hot Jupiter HD189733b: Constraints from the Reflection Spectrum," ApJ 786, 154 (May 2014).

[24] Scott, A. and Duley, W. W., "Ultraviolet and Infrared Refractive Indices of Amorphous Silicates," Astrophysical Journal Supplement 105, 401 (Aug. 1996).

[25] Barstow, J. K., Aigrain, S., Irwin, P. G. J., Bowles, N., Fletcher, L. N., and Lee, J.-M., "On the potential of the EChO mission to characterize gas giant atmospheres," MNRAS 430, 1188-1207 (Apr. 2013).

[26] McCullough, P. R., Crouzet, N., Deming, D., and Madhusudhan, N., "Water Vapor in the Spectrum of the Extrasolar Planet HD 189733b. I. The Transit," ApJ 791, 55 (Aug. 2014).

[27] Doyon, R., Hutchings, J. B., Beaulieu, M., Albert, L., Lafrenière, D., Willott, C., Touahri, D., Rowlands, N., Maszkiewicz, M., Fullerton, A. W., Volk, K., Martel, A. R., Chayer, P., Sivaramakrishnan, A., Abraham, R., Ferrarese, L., Jayawardhana, R., Johnstone, D., Meyer, M., Pipher, J. L., and Sawicki, M., "The JWST Fine Guidance Sensor (FGS) and Near-Infrared Imager and Slitless Spectrograph (NIRISS)," in [Space Telescopes and Instrumentation 2012: Optical, Infrared, and Millimeter Wave], Proceedings of SPIE 8442, 84422R (Sept. 2012).

[28] Ferruit, P., Birkmann, S., Böker, T., Sirriani, M., Giardino, G., Di Marchi, G., Alves de Oliveira, C., and Dorner, B., "Observing transiting exoplanets with JWST/NIRSpec," SPIE (2014).

[29] Kendrew, S., Scheithauer, S., Bouchet, P., Amiaux, J., Azzollini, R., Bouwman, J., Chen, C. H., Dubreuil, D., Fischer, S., Glasse, A., Greene, T. P., Lagage, P.-O., Lahuis, F., Ronayette, S., Wright, D., and Wright, G. S., "The Mid-Infrared Instrument for the James Webb Space Telescope, IV: The Low-Resolution Spectrometer," PASP 127, 623-632 (July 2015). 
[30] Deming, D., Seager, S., Winn, J., Miller-Ricci, E., Clampin, M., Lindler, D., Greene, T., Charbonneau, D., Laughlin, G., Ricker, G., Latham, D., and Ennico, K., "Discovery and Characterization of Transiting Super Earths Using an All-Sky Transit Survey and Follow-up by the James Webb Space Telescope," PASP 121, 952-967 (Sept. 2009).

[31] Pont, F., Sing, D. K., Gibson, N. P., Aigrain, S., Henry, G., and Husnoo, N., "The prevalence of dust on the exoplanet HD 189733b from Hubble and Spitzer observations," Monthly Notices of the Royal Astronomical Society 432, 2917-2944 (July 2013).

[32] Stevenson, K. B., Lewis, N. K., Bean, J. L., Beichman, C., Fraine, J., Kilpatrick, B. M., Krick, J. E., Lothringer, J. D., Mandell, A. M., Valenti, J. A., Agol, E., Angerhausen, D., Barstow, J. K., Birkmann, S. M., Burrows, A., Charbonneau, D., Cowan, N. B., Crouzet, N., Cubillos, P. E., Curry, S. M., Dalba, P. A., de Wit, J., Deming, D., Desert, J.-M., Doyon, R., Dragomir, D., Ehrenreich, D., Fortney, J. J., Garcia Munoz, A., Gibson, N. P., Gizis, J. E., Greene, T. P., Harrington, J., Heng, K., Kataria, T., Kempton, E. M.-R., Knutson, H., Kreidberg, L., Lafreniere, D., Lagage, P.-O., Line, M. R., Lopez-Morales, M., Madhusudhan, N., Morley, C. V., Rocchetto, M., Schlawin, E., Shkolnik, E. L., Shporer, A., Sing, D. K., Todorov, K. O., Tucker, G. S., and Wakeford, H. R., "Transiting Exoplanet Studies and Community Targets for JWST's Early Release Science Program," PASP (Feb. 2016). 